Article

\title{
An Optically Transparent Metasurface-Based Resonant Cavity Fed by Patch Antenna for Improved Gain
}

\author{
Qinlong $\mathrm{Li}^{1}{ }^{1}$, Xiaoming Chen ${ }^{1, *(\mathbb{D})}$ and $\mathrm{Xin}^{\mathrm{Hu}}{ }^{2, *}$ \\ 1 School of Information and Communications Engineering, Xi'an Jiaotong University, Xi'an 710049, China; \\ liq1519@mail.xjtu.edu.cn \\ 2 School of Electronic Engineering, Beijing University of Posts and Telecommunications, Beijing 100876, China \\ * Correspondence: xiaoming.chen@mail.xjtu.edu.cn (X.C.); huxin2016@bupt.edu.cn (X.H.)
}

Received: 21 September 2019; Accepted: 19 November 2019; Published: 20 November 2019

\begin{abstract}
An optically transparent metasurface (MS) is proposed to design a resonant cavity fed by a patch antenna operating at $5.6 \mathrm{GHz}$. In the proposed MS, a transparent micro metal mesh conductive (MMMC) film is used as the transparent conducting film (TCF), and it has a high optical transmittance of more than $75 \%$ and a low sheet resistance of $0.7 \Omega / \mathrm{sq}$. The MS is composed of a layer of glass substrate and a layer of MMMC film. The unit cell of MS consists of a square patch using MMMC film patterned on a square glass substrate. The transparent MS, patch antenna, ground plane, and air-filled half-wavelength cavity form a resonant cavity antenna, to achieve an improved gain. The MS is designed, optimized and analyzed using the EM simulation software CST. Results show that the MS can improve the simulated boresight gain from 4.7 to $13.2 \mathrm{dBi}$ by $8.5 \mathrm{~dB}$, without affecting the impedance bandwidth (IMBW) much. The losses of MS with different values of sheet resistance are also studied, showing the MS using MMMC with sheet resistance of $0.7 \Omega /$ sq has very small losses.
\end{abstract}

Keywords: metasurface; transparent conductive film; patch antenna; gain improvement

\section{Introduction}

In recent years there has been increased interest in thin-film, electromagnetically sensitive surfaces known as metasurfaces (MS). Metasurfaces are typically defined as a designed structures with lattice constants and thicknesses bellow the Bragg limit. Various kinds of MSs have been reported to improve the performances of antennas [1-8]. For example, due to the $0^{\circ}$ reflection phase of an artificial magnetic surface (AMC), the antenna can have a higher boresight gain with a lower profile by using AMC as ground plane below the radiating element, compared with perfect electrical conductor ground [1]. To enhance the gain of antenna, gradient-refractive index (GRIN) MS can be used for phase compensation to transform the quasi-sphere Electromagnetic (EM) wave radiated by an antenna to a near planar wave [2,3]. In reference [4], with a MS placed above a crossed slot circular polarized antenna, the bandwidth and gain could be increased. By using a MS above a horn antenna, the polarization can be rotated $90^{\circ}[5,6]$. In reference [7], the MS is used to change the linearly polarized EM waves from an antenna into circularly polarized EM waves. In reference [8], a radar cross-section (RCS) reduction technique by using the coding diffusion MS is presented, and the MS is optimized through a random optimization algorithm.

Recently, some microwave components, such as antennas and couplers, have been designed using transparent conductive film (TCF) for the purpose of aesthetics [9-13]. There are a lot of kinds of optically transparent and electrically TCFs manufactured by Cima Nano Tech company using self-assembling nanoparticle technology [9-11]. The sheet resistance of micro metal mesh conductive 
(MMMC) film is much lower than other TCFs such as indium tin oxide (ITO) [12], AgHT-8 and AgHT-4 [13]. Therefore, the microwave components using MMMC film will have less Ohmic loss, and this conductive film has already been applied in antenna [9], branch-line coupler [10], phase shifter [11].

In this paper, we propose an optically transparent MS using MMMC film to design a resonant cavity fed [14-17] by a patch antenna operating at $5.6 \mathrm{GHz}$. To the best knowledge of the authors, no one has used MMMC film to design a MS for the resonant cavity antenna [14-17]. The unit cell of MS consists of a square patch using MMMC film patterned on a square glass substrate. Two models, including a superstrate reflection model (SRM) and defect cavity model (DCM), are used to analyze the MS and the cavity, respectively. The proposed MS has a sufficiently strong reflectivity of more than 0.8 using SRM. However, the whole resonant cavity including the MS, ground plane, and air-filled half-wavelength cavity, has a passband at the resonant frequency of $5.6 \mathrm{GHz}$ using DCM. The losses of MS with different values of sheet resistance are also studied, indicating the MS using the proposed MMMC film also has very small losses. A patch antenna is used for studying the gain enhancement of the MS. Measurement results demonstrate that the proposed MS has little influence on the impedance bandwidth (IMBW) of the patch antenna, whereas it has the ability to improve the measured boresight gain by $7 \mathrm{~dB}$ (from 5.2 to $12.2 \mathrm{dBi}$ ) at the resonant frequency of $5.6 \mathrm{GHz}$.

\section{Antenna Design}

In our design, a transparent micro metal mesh conductive (MMMC) film is used as the transparent conducting film (TCF), which is constructed by Cima Nano Tech using SANTE self-assembling nanoparticle technology. It has a high optical transmittance of more than $75 \%$ and a low sheet resistance of $0.7 \Omega /$ sq. The MMMC film consists of two layers, a conductive layer made of silver laminated over a layer of polyethylene terephthalate (PET). Figure 1 shows the transparent MMMC film viewed through microscope. It can be seen that conductive strips are distributed on the PET layer randomly. The optical transparency of the mesh material is quantitatively calculated by the percentage of non-metal strips area to the total area of the material, which is given as Equation (1). With the increase of non-metal strips area, the transparency will increase:

$$
\text { Optical transparency }=\frac{A_{\text {Non-metal }}}{A_{\text {total }}} \cdot 100 \%
$$

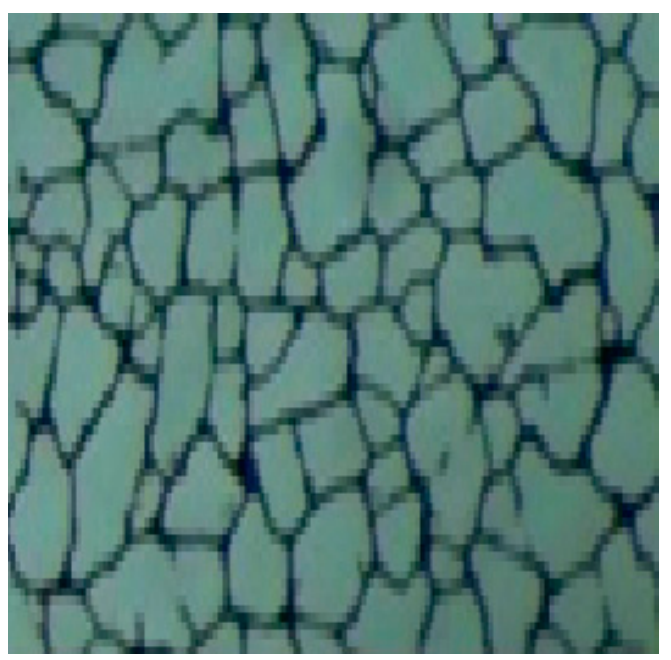

Figure 1. MMMC film viewed through microscope.

The configuration of designed MS is shown in Figure 2, where 81 unit cells are placed periodically in a $9 \times 9$ layout as shown in Figure 2a, with a total size of $W s \times W s=81 \times 81 \mathrm{~mm}^{2}$. Each unit cell is a square patch using MMMC film patterned on a square glass substrate with a relative permittivity of 5.7, 
as shown in Figure 2b. The PET has a relative permittivity of 5.1. To verify the gain improvement of the designed MS, a patch antenna shown in Figure $3 \mathrm{a}$ is proposed. It has a planar structure, comprising a square substrate, a rectangular radiating patch at the top side of substrate and a square metallic ground at the bottom side. The total dimension of the patch antenna is $\mathrm{Ws} \times \mathrm{Ws}=81 \times 81 \mathrm{~mm}^{2}$. The substrate is made of Rogers 4350B, with a thickness of $0.8 \mathrm{~mm}$, a relative permittivity of 3.5 and a loss tangent of 0.004 . To feed the antenna, a coaxial cable is used with inner pin connected to the radiating patch via a hole through the Rogers substrate, while the metal outside of coaxial cable is soldered on the metallic ground. As shown in Figure 3b, the MS is above the patch antenna for gain improvement at a height of $h$, forming MS antenna. Parameters of the MS antenna are listed in Table 1 and used for fabrication of the prototype. In the fabrication process of MS, we used the LPKF prototype machine to cut lines on the MMMC film, and then used a knife to scrape the unnecessary parts. In the prototype, four nylon posts at four corners are deployed to support the height between the patch antenna and the MS for measurement. The fabricated MS, patch antenna, and MS antenna are shown in Figure 4.

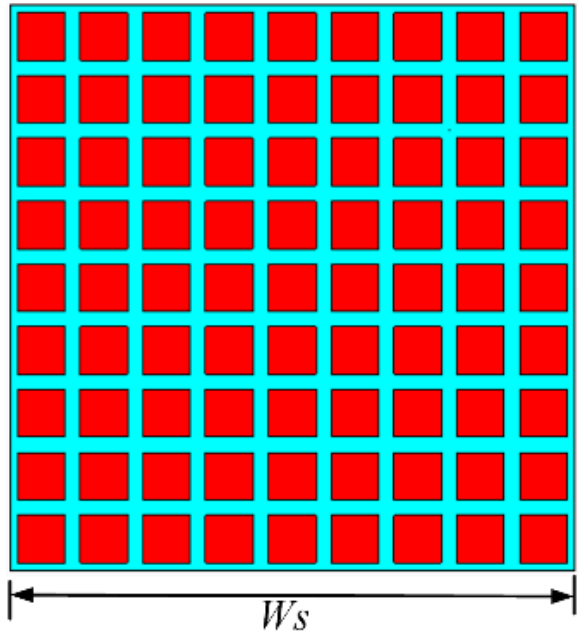

(a)

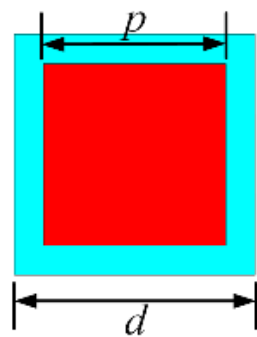

(b)

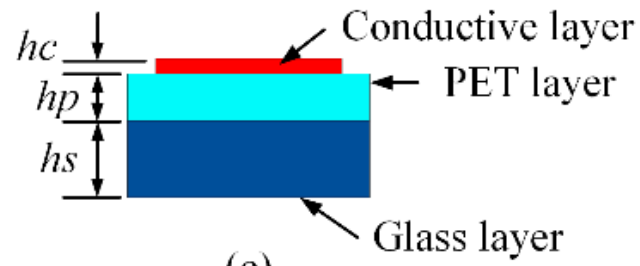

(c)

Figure 2. Proposed MS: (a) top view of MS, (b) top view of unit cell, and (c) side view of unit cell.

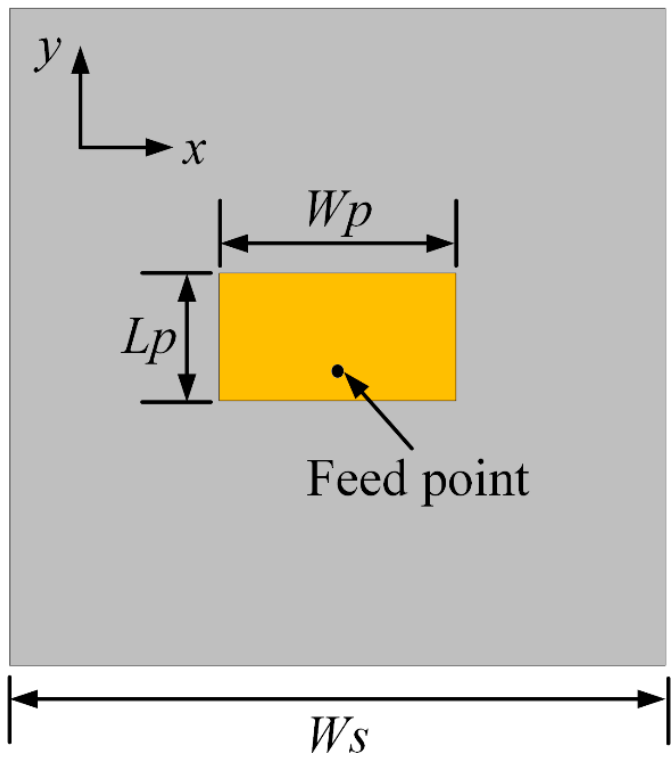

(a)

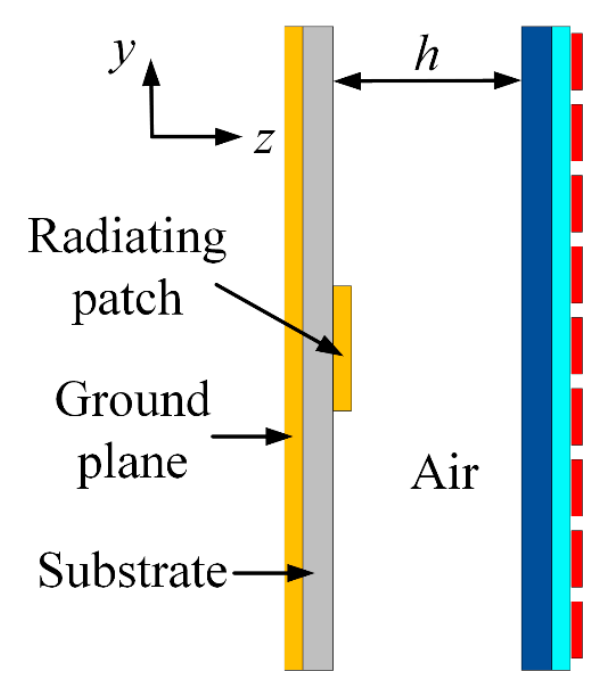

(b)

Figure 3. (a) The geometry of patch antenna (b) MS antenna. 


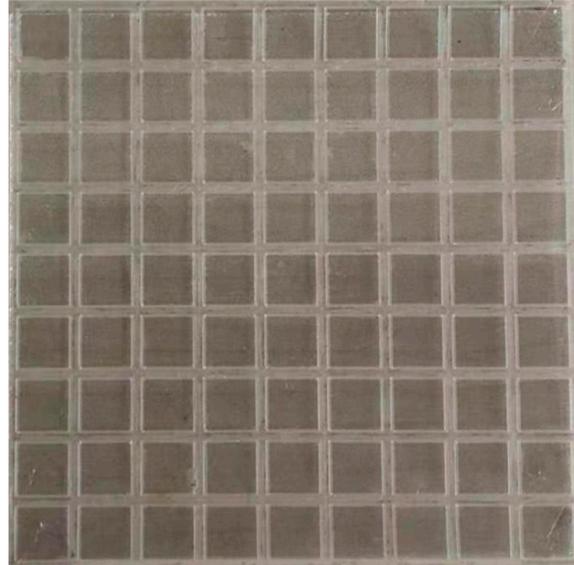

(a)

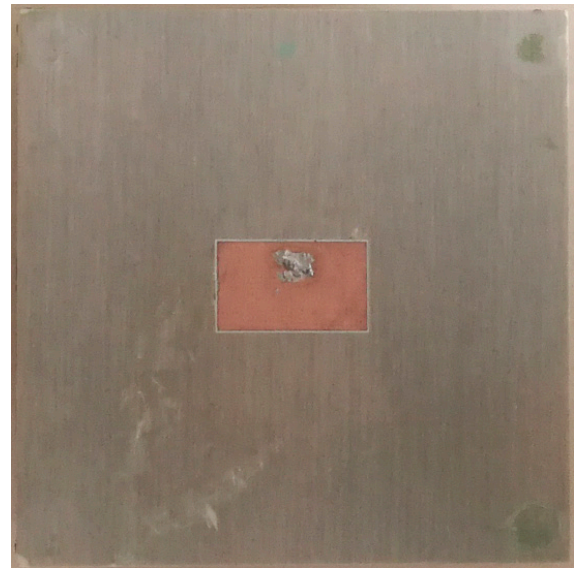

(b)

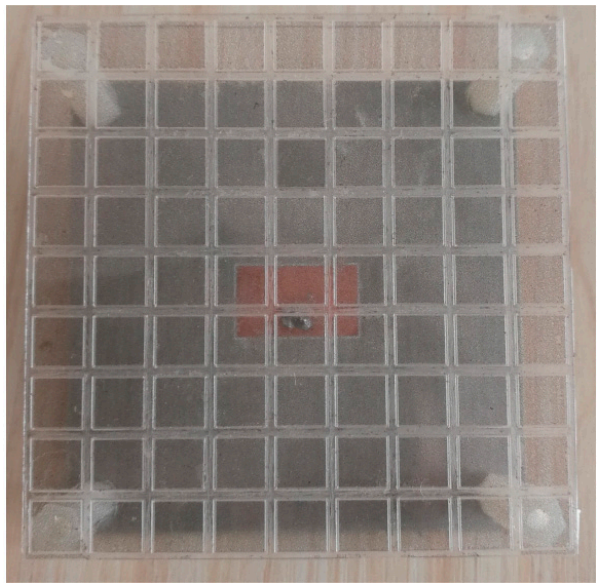

(c)

Figure 4. Prototype of (a) MS (b) patch antenna (c) MS antenna.

Table 1. Dimensions of the proposed antenna (unit: $\mathrm{mm}$ ).

\begin{tabular}{ccccccccc}
\hline$W_{s}$ & $W_{p}$ & $L_{p}$ & $p$ & $d$ & $h$ & $h c$ & $h p$ & $h s$ \\
\hline 81 & 22 & 13 & 9 & 6.8 & 29.5 & 0.005 & 0.012 & 1 \\
\hline
\end{tabular}

\section{MS Study}

\subsection{Operation Principle}

In the proposed design, the transparent MS has a characteristic of partial but high reflection, acting as a partially reflective surface (PRS). Thus, the MS antenna in Figure 3b including the patch antenna, MS, ground plane, and air-filled half-wavelength cavity, can be regarded as a resonant cavity antenna [14-17]. In this antenna, the EM waves emitted from patch antenna undergo multiple reflections and transmissions within the air-filled cavity between the metallic ground and MS. When all the waves transmitted through the PRS are in phase [18], the antenna will have the maximum gain in boresight direction. An antenna's directivity can be enhanced significantly using the multiple reflections between the ground plane and the PRS. The operating frequency of the resonant cavity antenna can be expressed on the following equation [18]:

$$
f=\frac{c}{4 \pi h}\left(\varphi_{p r s}+\pi-2 n \pi\right), n=0,1,2 \ldots
$$

where $h$ is the cavity height, $\varphi_{p r s}$ is the reflection phase of the PRS and $\pi$ is the reflection phase of the ground plane. 
The proposed MS can be analyzed using superstrate reflection model (SRM) and defect cavity model (DCM) as follows $[19,20]$. In order to obtain high gain of antenna, the MS should have sufficiently strong reflectivity (0.6-0.9) [21]. The reflection magnitude of the proposed MS can be calculated using SRM depicted in Figure 5a, by using the template of frequency-selective surface (FSS) in CST. The unit cell in Figure $5 a$ has the same size as in Figure $2 b$,c. After applying linearly polarized plane EM wave incident from port 1 , the $S_{11}$ and $S_{21}$ between two ports can be calculated by using periodic boundary in the model. The reflection and transmission magnitude for SRM are also measured. It can be seen that both the simulated and measured reflection magnitude $\left|\Gamma_{\mathrm{SRM}}\right|$ of the proposed MS is about 0.8 at the desired resonant frequency of $5.6 \mathrm{GHz}$, as shown in Figure $5 b$, satisfying the requirement for PRS within $0.6-0.9$.

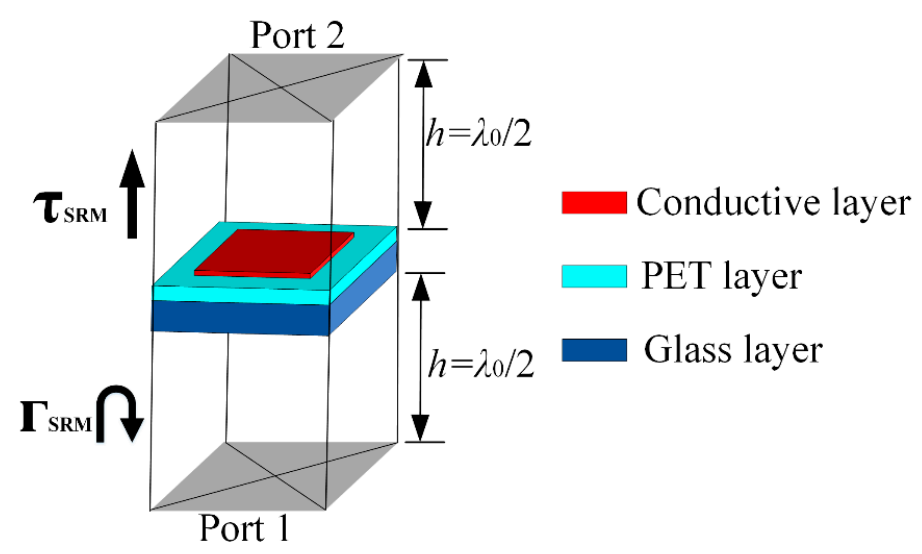

(a)

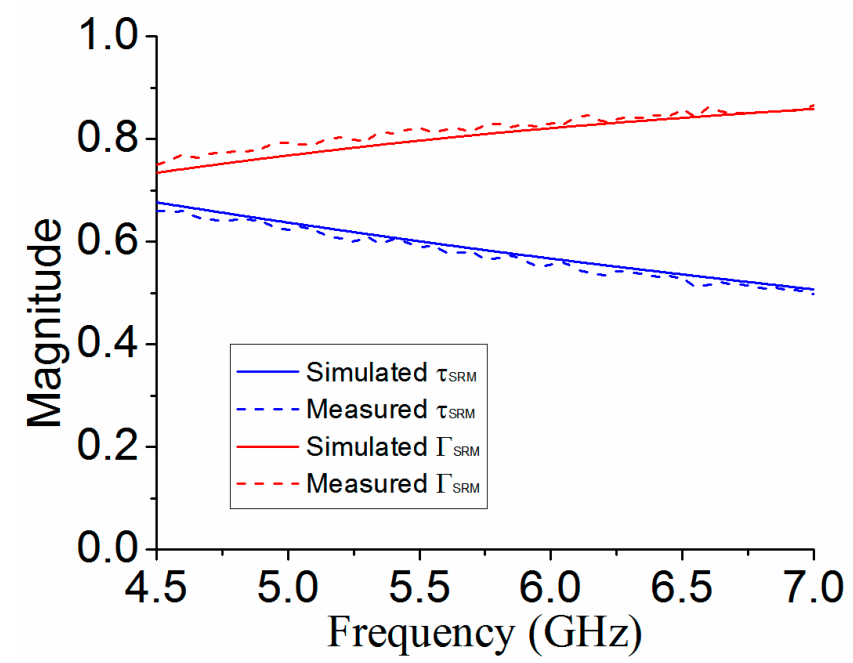

(b)

Figure 5. (a) Unit cell of the MS using SRM (b) simulated and measured reflection and transmission magnitude.

Different from SRM to evaluate the reflection magnitude of MS, DCM is developed to study the whole resonant cavity including the MS, ground plane, and air-filled half-wavelength cavity [22]. The equivalent unit cell of the resonant cavity can be analyzed using mirror image theory as shown in Figure 6a. The metallic ground plane generates the electric image of the MS, and the unit cell of the resonant cavity consists of the unit cell of MS and its image through the symmetry plan. As the same as the unit cell of MS, the S-parameters between ports 1 and 2 of the unit cell of the resonant cavity are also calculated using periodic boundary, and the simulation results are shown in Figure $6 \mathrm{~b}$. Although the MS alone has a high reflectivity, a passband is generated at the resonant frequency of 
$5.6 \mathrm{GHz}$ for the whole resonant cavity. The transmission bandwidth with $\left|\tau_{\mathrm{DCM}}\right| \geq 0.7$ is $5.4-5.8 \mathrm{GHz}$, indicating that most of the EM power in the cavity can be transmitted through the MS in this frequency band [21]. For measurement, the measured reflection and transmission magnitude for DCM have good agreements with simulation results. With the height $h=29.5 \mathrm{~mm}$, thickness $h p=0.012 \mathrm{~mm}$ and $h s=1 \mathrm{~mm}$ unchanged, the desired resonant frequency of the cavity is achieved by tuning the length $p$ and $d$ of the unit cell. In our design, $d=9 \mathrm{~mm}$ and $p=6.8 \mathrm{~mm}$ are selected.

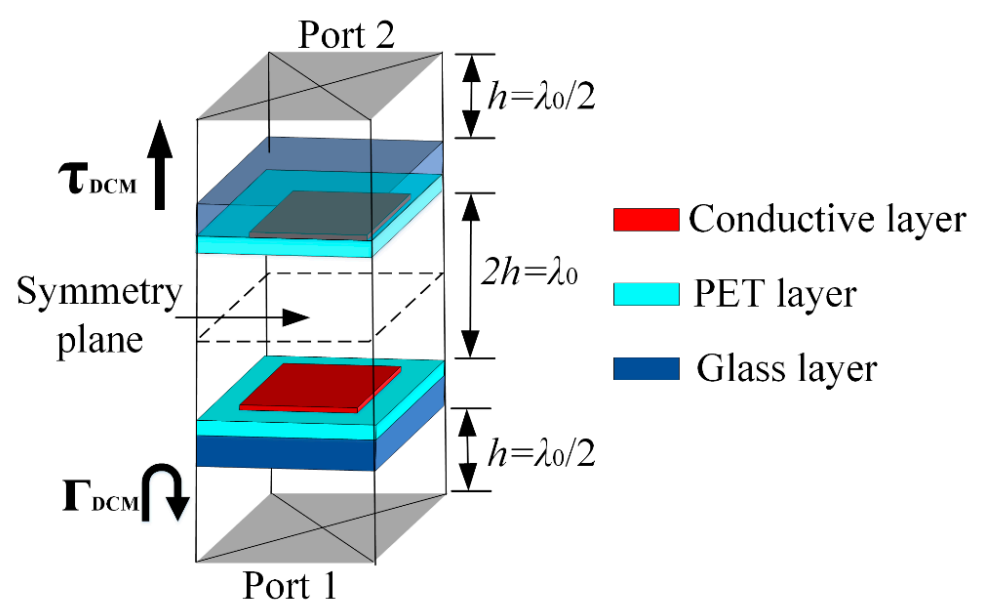

(a)

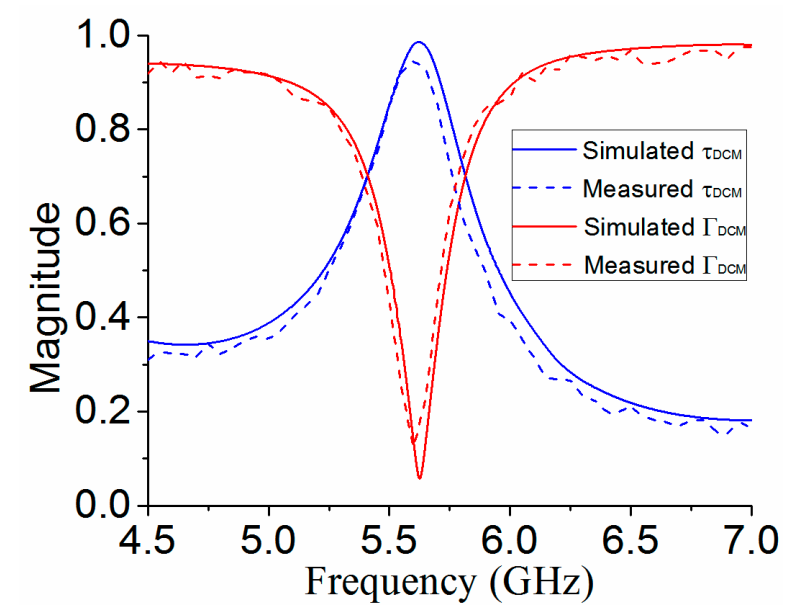

(b)

Figure 6. (a) Unit cell of the resonant cavity using DCM (b) simulated and measured reflection and transmission magnitude.

\subsection{Effects of Sheet Resistance}

The TCF used for the design of MS is an MMMC film. The conductive layer of MMMC film in Figure 1 has a sheet resistance of $0.7 \Omega / \mathrm{sq}$, and a thickness of $0.005 \mathrm{~mm}$. It is essential to study the losses of MS by using the MMMC film. A parametric study has been carried out using simulation to study the effect of sheet resistance on the losses of MS. The DCM is used to analyze the losses. With the different values of sheet resistance, and a thickness remaining unchanged at $0.005 \mathrm{~mm}$, the reflection magnitude $\left|\Gamma_{\mathrm{DCM}}\right|$ and transmission magnitude $\left|\tau_{\mathrm{DCM}}\right|$ can be computed by simulation in CST, and the results are shown in Figure 7. The losses of MS in the cavity can be calculated using Equation (3). Thus, using the results in Figure 7, the losses of MS with different values of sheet resistance in the cavity are calculated as shown in Figure 8. It can be seen that, with the increase of sheet resistance, the losses become larger. In our design, the conductive layer of MMMC film has a sheet resistance of $0.7 \Omega / s q$, and the losses are less than 0.03 , as shown in Figure 8, which are very small. The results with sheet 
resistance less than $0.7 \Omega / \mathrm{sq}$ are nearly unchanged compared with the results with sheet resistance of $0.7 \Omega /$ sq, so they are not shown for brevity.

$$
\text { Losses }=1-\left|\tau_{\mathrm{DCM}}\right|^{2}-\left|\Gamma_{\mathrm{DCM}}\right|^{2}
$$

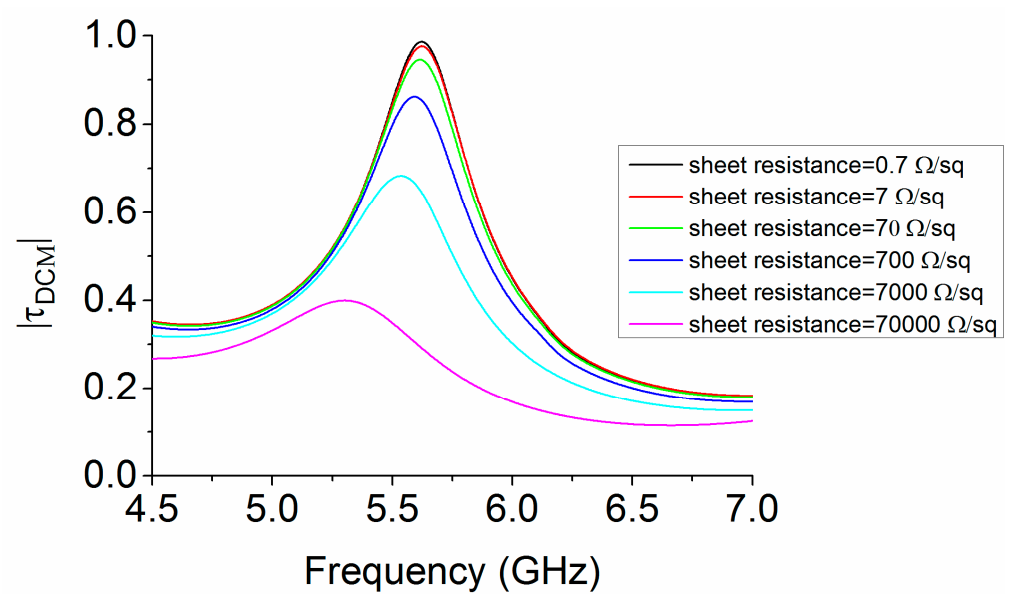

(a)

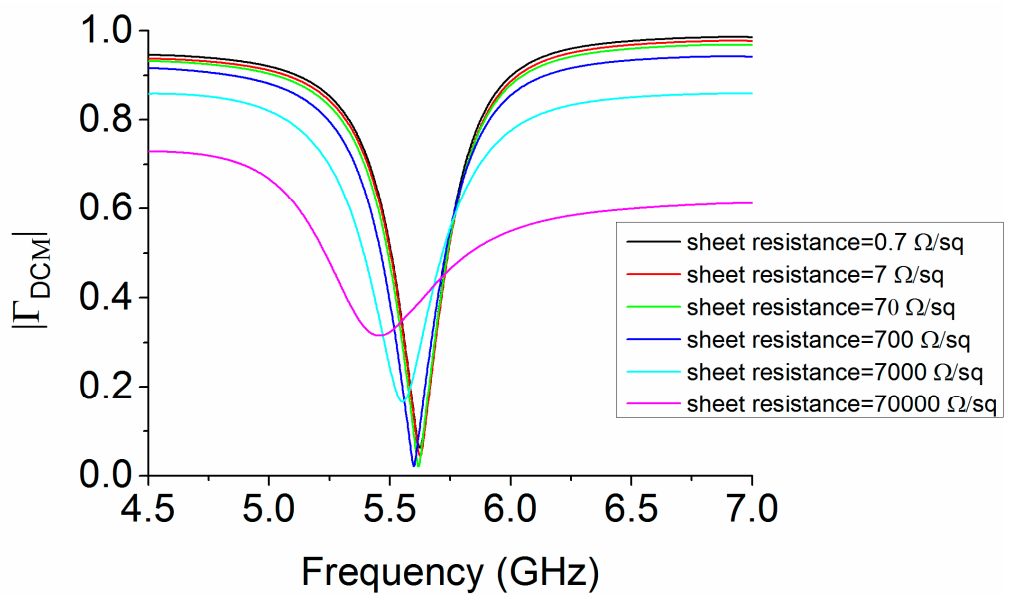

(b)

Figure 7. Computed reflection and transmission magnitude using different values of sheet resistance (a) $\left|\tau_{\mathrm{DCM}}\right|(\mathbf{b})\left|\Gamma_{\mathrm{DCM}}\right|$.

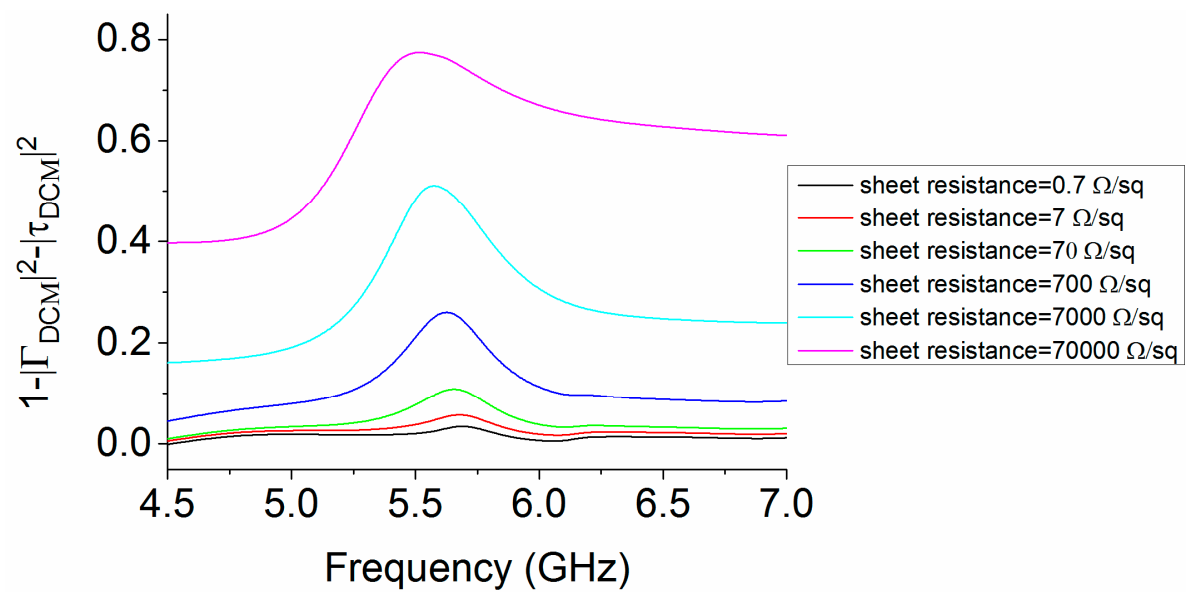

Figure 8. Losses of MS with different values of sheet resistance. 


\subsection{Comparison with Copper}

The conductivity of the MMMC is high, but it is still orders of magnitude worse than a material like copper. Therefore, it is significant to compare the results of MS if the transparent conductive layer in the MS is replaced by copper. The copper's conductivity in the simulation is $5.8 \times 10^{7} \mathrm{~S} / \mathrm{m}$, with the same thickness of $0.05 \mathrm{~mm}$, so the equivalent sheet resistance can be calculated as $0.0034 \Omega / \mathrm{sq}$. The results are shown in Figure 9. It can be seen that there is little difference for the performances of MS for both SRM and DCM when the MMMC film is replaced by copper.

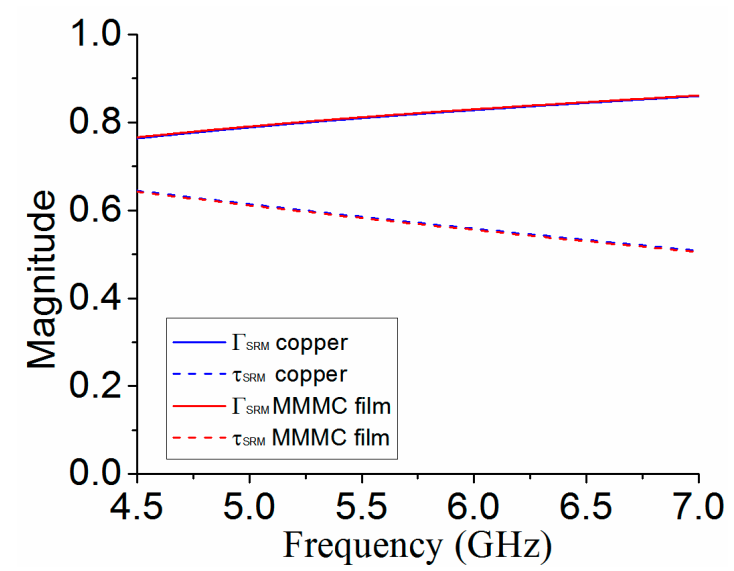

(a) SRM

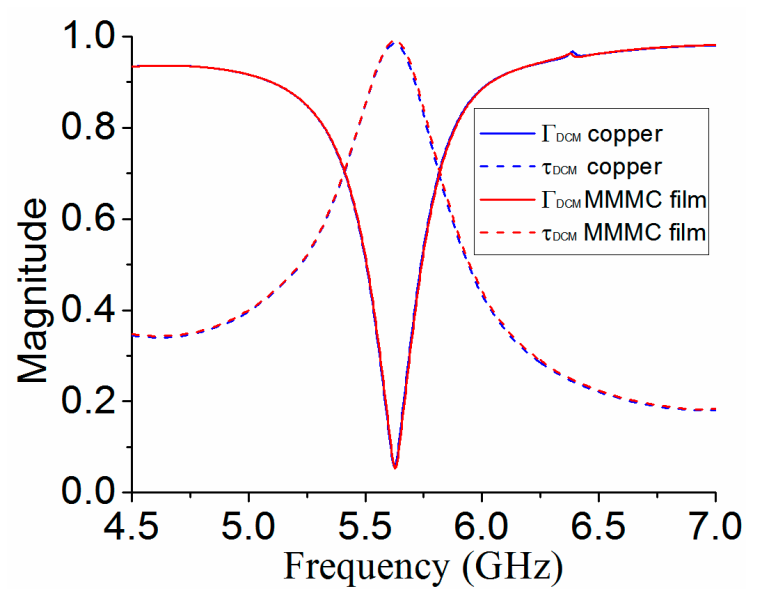

(b) DCM

Figure 9. Performances comparison of MS between using MMMC film and copper.

\section{Simulation and Measurement Results}

The full-wave simulation tool CST is used to carry out simulations for optimization and analysis of both patch and MS antenna in Figure 3a,b. For verification, the fabricated antennas are measured by a vector-network analyzer (VNA) for $S_{11}$ and the antenna measurement system Satimo Starlab for boresight gains and radiation patterns. The simulation and measurement results for both patch antenna and MS antenna have good agreement. Figure 10 shows the simulated and measured $S_{11}$ for both MS antenna and patch antenna. The simulated impedance bandwidths (IMBWs) with $S_{11}<-10 \mathrm{~dB}$ for the MS antenna and patch antenna are 5.53-5.67 GHz and 5.55-5.67, respectively. The measured IMBWs are $5.52-5.66 \mathrm{GHz}$ and $5.56-5.69 \mathrm{GHz}$ for both the MS antenna and patch antenna. Therefore, the use of MS for antenna gain improvement has little influence on the IMBW of the patch antenna. 


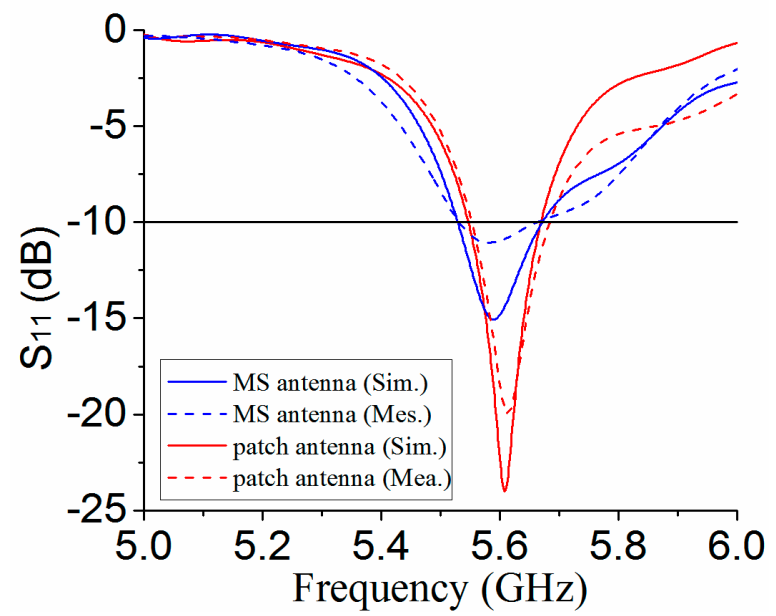

Figure 10. Simulated and measured $S_{11}$ for patch antenna and MS antenna.

Figure 11 shows the simulated and measured boresight gains for the MS antenna and patch antenna. It can be seen that the MS can improve the boresight gains from $4.7 \mathrm{dBi}$ to $13.2 \mathrm{dBi}$ for simulation, while it can enhance boresight gains from 5.2 to $12.2 \mathrm{dBi}$ for measurement at the resonant frequency of $5.6 \mathrm{GHz}$. Figure 12 shows the simulated and measured boresight efficiencies for the MS antenna and patch antenna. The results show that simulated efficiencies of both patch antenna and MS antenna are more than $85 \%$ at $5.6 \mathrm{GHz}$, while the measured efficiencies are more than $80 \%$ at $5.6 \mathrm{GHz}$. These results also indicate the use of MS has very small losses.

Figure 13 shows the simulated and measured radiation patterns of the MS antenna and patch antenna at $5.6 \mathrm{GHz}$ in both z-x and z-y planes. In Figure 13a, it can be found that the patch antenna has half-power beam widths (HPBWs) of $68^{\circ}$ and $69.8^{\circ}$ in the z-x plane for both simulation and measurement, respectively. The MS antenna has much narrower HPBWs of $32^{\circ}$ and $33^{\circ}$ in z-x plane for both simulation and measurement. Similarly, the MS can also reduce the HPBWs significantly in z-y plane. It can be seen in Figure 13b that the patch antenna has the simulated and measured half-power beam widths (HPBWs) of $116.4^{\circ}$ and $122.7^{\circ}$ in z-y plane, respectively. However, the simulated and measured HPBWs of MS antenna are $35.4^{\circ}$ and $36.5^{\circ}$, much less than the corresponding results of patch antenna in z-y plane. These results indicate that, with the use of MS, the HPBWs can be reduced significantly, hence improving the boresight gains of patch antenna.

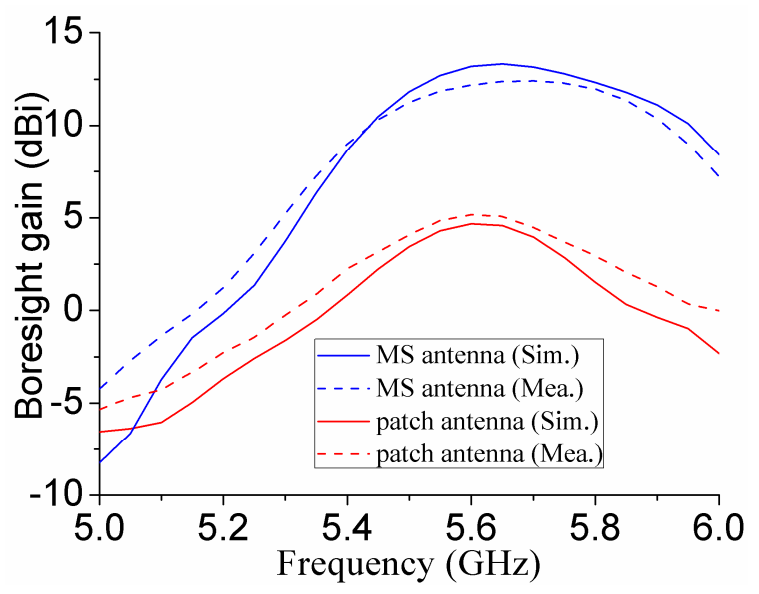

Figure 11. Simulated and measured boresight gains for patch antenna and MS antenna. 


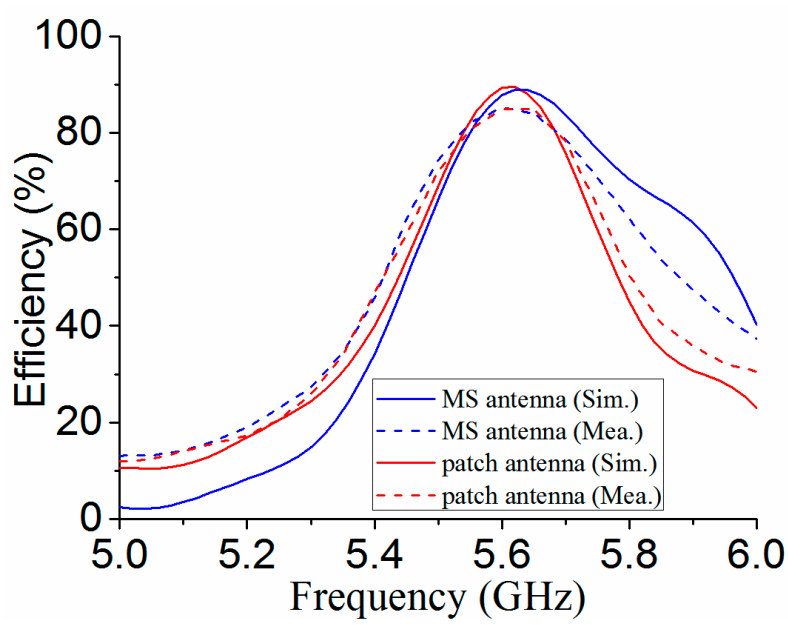

Figure 12. Simulated and measured efficiencies for patch antenna and MS antenna.

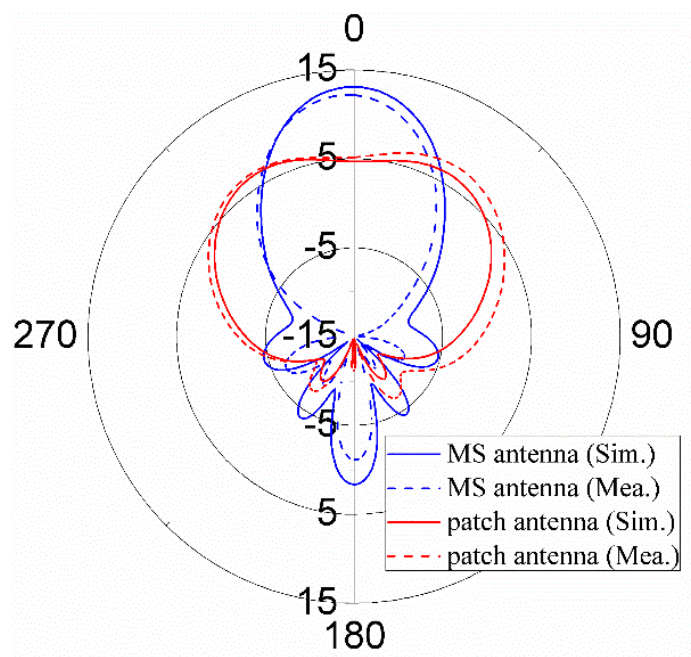

(a) z-x plane

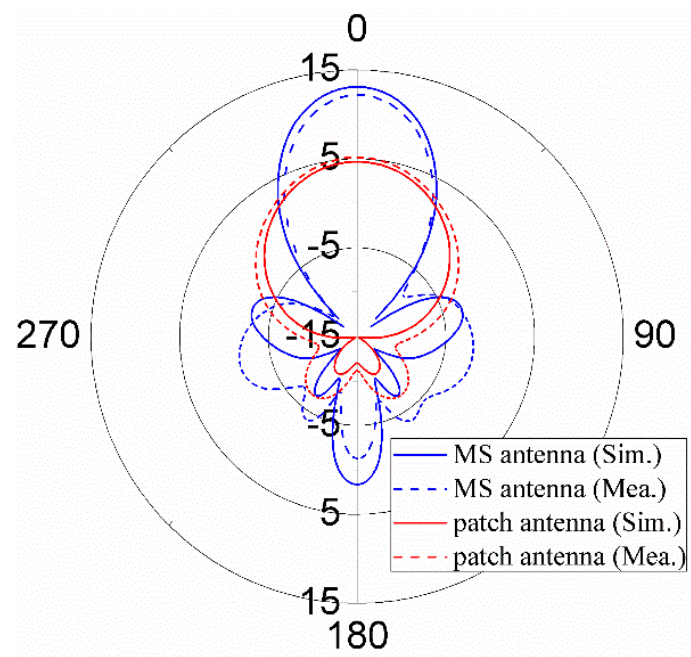

(b) z-y plane

Figure 13. Simulated and measured radiation patterns.

\section{Conclusions}

An optically transparent MS using MMMC film is proposed to design a resonant cavity fed by a patch antenna. The TCF used in the proposed MS is MMMC film, with a low sheet resistance of $0.7 \Omega /$ sq and a highly optically transmittance more than $75 \%$. The unit cell of MS consists of a square patch using MMMC film patterned on a square glass substrate. Two models, including SRM and DCM, are used to analyze the operation principle of the MS. The losses of MS with different values of sheet resistance are also studied, showing the MS using MMMC with sheet resistance of $0.7 \Omega / \mathrm{sq}$ has very small losses. Measurement results show that the proposed MS antenna and patch antenna have similar IMBWs, but the MS antenna has a much higher gain compared with the patch antenna.

Author Contributions: Conceptualization, Q.L. and X.C.; Formal analysis, Q.L., X.C. and X.H.; Methodology, Q.L., X.C. and X.H.; Software, Q.L. and X.H.; Validation, Q.L.; Writing-original draft, Q.L. and X.C.

Funding: This work was supported in part by the National Natural Science Foundation of China under Grant 61801366 and in part by the China Postdoctoral Science Foundation under Grant 2019TQ0258.

Conflicts of Interest: The authors declare no conflict of interest. 


\section{References}

1. Li, G.; Zhai, H.; Li, L.; Liang, C.; Yu, R.; Liu, S. AMC-Loaded Wideband Base Station Antenna for Indoor Access Point in MIMO System. IEEE Trans. Antennas Propag. 2015, 2, 525-532. [CrossRef]

2. Sayanskiy, A.; Glybovski, S.; Akimov, V.P.; Filonov, D.; Belov, P.; Meshkovskiy, I. Broadband 3-D Luneburg Lenses Based on Metamaterials of Radially Diverging Dielectric Rods. IEEE Antennas Wirel. Propag. Lett. 2017, 16, 1520-1523. [CrossRef]

3. Brocker, D.E.; Turpin, J.P.; Werner, P.L.; Werner, D.H. Optimization of Gradient Index Lenses Using Quasi-Conformal Contour Transformations. IEEE Antennas Wirel. Propag. Lett. 2014, 13, 1787-1791. [CrossRef]

4. Zhou, C.F.; Cheung, S.W.; Li, Q.L.; Li, M. Bandwidth and gain improvement of a crossed slot antenna with metasurface. Appl. Phys. Lett. 2017, 110, 211603. [CrossRef]

5. Saikia, M.; Ghosh, S.; Srivastava, K.V. Design and Analysis of Ultrathin Polarization Rotating Frequency Selective Surface Using V-Shaped Slots. IEEE Antennas Wirel. Propag. Lett. 2017, 16, 2022-2025. [CrossRef]

6. Li, Y.; Cao, Q.S.; Wang, Y. A Wideband Multifunctional Multilayer Switchable Linear Polarization Metasurface. IEEE Antennas Wirel. Propag. Lett. 2018, 18, 1314-1318. [CrossRef]

7. Zhu, H.L.; Cheung, S.W.; Chung, K.L.; Yuk, T.I. Linear-to-Circular Polarization Conversion Using Metasurface. IEEE Trans. Antennas Propag. 2013, 61, 4615-4623. [CrossRef]

8. Ali, L.; Li, Q.L.; Khan, T.A.; Yi, J.; Chen, X. Wideband RCS Reduction Using Coding Diffusion Metasurface. Material 2019, 12, 2708. [CrossRef]

9. Li, Q.L.; Cheung, S.W.; Wu, D.; Yuk, T.I. Optically Transparent Dual-Band MIMO Antenna Using Micro-Metal Mesh Conductive Film for WLAN System. IEEE Antennas Wirel. Propag. Lett. 2017, 16, 920-923. [CrossRef]

10. Sa'ad, B.M.; Rahim, S.K.A.; Peter, T.; Rani, M.S.B.A.; Ausordin, S.F.; Zaidel, D.N.A.; Krishnan, C. Transparent Branch-Line Coupler Using Micro-Metal Mesh Conductive Film. IEEE Microw. Wirel. Compon. Lett. 2014, 24, 857-859. [CrossRef]

11. Sa'ad, B.M.; Rahim, S.K.A.; Peter, T. Transparent $0^{\circ}$ phase shifter using micro-metal mesh conductive film. Electron. Lett. 2015, 51, 841-843. [CrossRef]

12. Serra, C.C.; Medeiros, C.R.; Costa, J.R.; Fernandes, C.A. Mirror-Integrated Transparent Antenna for RFID Application. IEEE Antennas Wirel. Propag. Lett. 2011, 10, 776-779. [CrossRef]

13. Hakimi, S.; Rahim, S.K.A.; Abedian, M.; Noghabaei, S.M.; Khalily, M. CPW-Fed Transparent Antenna for Extended Ultrawideband Applications. IEEE Antennas Wirel. Propag. Lett. 2014, 13, 1251-1254. [CrossRef]

14. Zeb, B.A.; Hashmi, R.M.; Esselle, K.P. Wideband gain enhancement of slot antenna using one unprinted dielectric superstrate. Electron. Lett. 2015, 51, 1146-1148. [CrossRef]

15. Yahiaoui, R.; Burokur, S.N.; Lustrac, A. Enhanced directivity of ultra-thin metamaterial-based cavity antenna fed by multisource. Electron. Lett. 2009, 45, 814-816. [CrossRef]

16. Burokur, S.N.; Yahiaoui, R.; Lustrac, A. Subwavelength metamaterial-based resonant cavities fed by multiple sources for high directivity. Microw. Opt. Techn. Lett. 2009, 51, 1883-1888. [CrossRef]

17. Yahiaoui, R.; Burokur, S.N.; Vigneras, V.; Lustrac, A.; Mounaix, P. Investigation of spatial filters at microwave frequencies: Application for antenna directivity enhancement. Microw. Opt. Techn. Lett. 2012, 54, 1327-1332. [CrossRef]

18. Trentini, G.V. Partially Reflecting Sheet Arrays. IRE Trans. Antennas Propag. 1956, 4, 666-671. [CrossRef]

19. Ge, Y.; Esselle, K.P.; Bird, T.S. The Use of Simple Thin Partially Reflective Surfaces with Positive Reflection Phase Gradients to Design Wideband, Low-Profile EBG Resonator Antennas. IEEE Trans. Antennas Propag. 2012, 60, 743-750. [CrossRef]

20. Thevenot, M.; Cheype, C.; Reineix, A.; Jecko, B. Directive Photonic-Bandgap Antennas. IEEE Trans. Microw. Theory Tech. 1999, 47, 2115-2122. [CrossRef]

21. Feresidis, A.P.; Vardaxoglou, J.C. High gain planar antenna using optimised partially reflective surfaces. IET Microw. Antennas Propag. 2001, 148, 345-350. [CrossRef]

22. Hashmi, R.M.; Zeb, B.A.; Esselle, K.P. Wideband High-Gain EBG Resonator Antennas with Small Footprints and All-Dielectric Superstructures. IEEE Trans. Antennas Propag. 2014, 62, 2970-2977. [CrossRef]

(C) 2019 by the authors. Licensee MDPI, Basel, Switzerland. This article is an open access article distributed under the terms and conditions of the Creative Commons Attribution (CC BY) license (http://creativecommons.org/licenses/by/4.0/). 